\title{
Exploring the Mechanisms of Anti-A $\beta 42$ Aggregation Activity of Walnut-derived Peptides using Transcriptomics and Proteomics in vitro
}

\author{
Min Wang ${ }^{\circledR 1}$, Congcong Gong ${ }^{\oplus 1}$, William Kwame Amakye ${ }^{\circledR 1}$, Jiaoyan Ren ${ }^{\circledR 1, *}$ \\ ${ }^{1}$ School of Food Science and Engineering, South China University of Technology, Wushan, 510641, Guangzhou, China
}

A R T I C L E I N F O
Article History
Received 19 November 2021
Accepted 14 December 2021
Keywords
transcriptomic
proteomic
Alzheimer's diseases
A $\beta 42$ aggregation
cell model
walnut-derived peptides.

ARTICLE INFO

Article History

Received 19 November 2021

Accepted 14 December 2021

Keywords

Alzheimer's diseases

cell model

walnut-derived peptides

\begin{abstract}
A B S T R A C T
Inhibiting $\beta$-amyloid $(\mathrm{A} \beta$ ) aggregation is of significance in finding potential candidates for Alzheimer's disease (AD) treatment. Accumulating evidence suggests that nutrition is important for improving cognition and reducing AD risk. Walnut has been widely used as a functional food for brain health; however the underlying mechanisms remain unknown. Here, we investigated the molecular level alteration in Arctic mutant $\mathrm{A} \beta 42$ induced aggregation cell model by RNA-seq and iTRAQ approaches after walnutderived peptides Pro-Pro-Lys-Asn-Trp (PW5) and Trp-Pro-Pro-Lys-Asn (WN5) interventions. PW5 or WN5 could significantly decrease abnormal $\mathrm{A} \beta 42$ aggregates. However, resultant alterations in transcriptome (substantially unchanged) were inconsistent with proteomic data (marked change). Proteomic analysis revealed 184 and 194 differentially expressed proteins unique to PW5 and WN5 treatment, respectively, for inhibiting $\mathrm{A} \beta 42$ protein production or increasing protein degradation via the mismatch repair pathways. Our study provides new insights into the effectiveness of food-derived peptides for anti-A $\beta 42$ aggregation in AD.
\end{abstract}

(C) 2021 The Authors. Publishing services by Visagaa Publishing House This is an open access article distributed under the CC BY-NC 4.0 license (https://creativecommons.org/licenses/by/4.0/).

\section{INTRODUCTION}

To date, omics studies such as transcriptomic and proteomics, as well as other high-throughput screening methods, that have been used to investigate changes at the molecular level in the response to environmental perturbations liking $\mathrm{pH}$, temperature, interferences, etc $[1,2]$. Although transcirptomics and proteomics reflect the different functional processes in molecular level, they are closely related but not always well matched. Not all changes in gene expression are necessarily reflected in the proteome, and vice versa [3]. In this scenario, combination of transcriptomics and proteomics approaches was the recommended method to study the mechanism under certain condition might provide comprehensive information during the biological processes in cells, including posttranscriptional translation [4,5]. Numerous biomedical studies have used these strategies to discover candidate gene or protein biomarkers for various diseases, as well as other studies in the areas related to life activities.

Alzheimer's disease (AD) is a severe and progressive neurodegenerative disease, whose condition of the brain that leading to the loss of memory and cognitive ability. In the past several decades, scientists' knowledge of the biology that underlies $\mathrm{AD}$ has advanced tremendously. Especially, the role of the sticky amyloid $\beta$-peptide $(\mathrm{A} \beta$ ) prominent in the brain plaques hallmark of $\mathrm{AD}$ is now much better understood [6]. It is known that mutations in the gene such as $\beta$-amyloid precursor protein (APP), presenilin-1 (PS1) and PS2, involving APP metabolism could cause $\mathrm{A} \beta$ plaques [7]. Growing evidences indicates that imbalance of $\mathrm{A} \beta$ metabolism including $\mathrm{A} \beta$ production and $\mathrm{A} \beta$ clearance resulted in $\mathrm{A} \beta$ aggregation around neurons. However, recent studies suggested that intracellular $\mathrm{A} \beta$ accumulation also has pathogenic relevance. Previous study reported that hereditary arctic mutation of $\mathrm{A} \beta 42$ $(\mathrm{E} 22 \mathrm{G})$ as a critical causative product in the pathology of $\mathrm{AD}$ that could lead to increased intracellular aggregation of $\mathrm{A} \beta$ in early-onset $\mathrm{AD}$ [8]. Lu et al. found that a single residue changes in the mutant $\mathrm{A} \beta 42$ peptide (E22G) led to significant accumulation of intracellular $\mathrm{A} \beta 42$ protein by constructing a cell model expressing mCherry-tagged E22G A $\beta 42$, which provides a platform for screening the potential anti- $\mathrm{A} \beta$ aggregation substances [9].

Emerging evidence suggests that nutrition is important for brain health and can positively impact on cognition and AD risk [10]. Walnut has long been widely used as a functional food for brain health due to its bioactive components such as unsaturated fatty acids, vitamins and proteins [11, 12]. In comparison with walnut protein, walnut-derived peptides have attracted much attention due to its low molecular weight and higher absorption rate [13]. Lots of bioactive peptides, including Trp-Asp-Gln-Trp (WW4), ProPro-Lys-Asn-Trp (PW5), Trp-Pro-Pro-Lys-Asn (WN5), Trp-SerArg-Glu-Glu-Gln-Glu (WE7), have been successfully separated and identified from walnut protein hydrolysates $[12,14,15]$. In our previous work, we found that WW4 and PW5 could effectively

${ }^{\star}$ Corresponding author. Email: jyren@scut.edu.cn

Peer review under responsibility of the International Association of Dietetic Nutrition and Safety

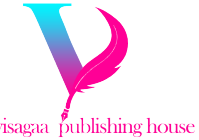


inhibit intracellular $\mathrm{A} \beta 42$ aggregation using the above-mentioned cell model $[12,14]$. Moreover, PW5 has been proven be capable of improving cognitive impairments in APP/PS1 transgenic mice through reducing $\mathrm{A} \beta$ deposition [12]. This indicated that this HEK293-E22G cell line could be a valid cell model for the screening of potential anti-aggregation substances for $\mathrm{AD}$ treatment. However, the aggregates inside cells and molecular changes behind the mutant $\mathrm{A} \beta 42$ peptide induced protein abnormal aggregation are still unknown.

Therefore, we firstly investigated the alterations in the RNA and proteins after transfecting the hereditary Arctic mutation of $\mathrm{A} \beta 42$ (E22G) plasmids by RNA-seq and iTRAQ approaches to measure identity and its expression level in cells. Also, we explored the changes in the genes and proteins of E22G cell model after walnutderived peptides PW5 and WN5 (which consisted of the same amino acids of PW5 but the sequence is different) treatments to elucidate the potential mechanism of anti-A $\beta 42$ aggregation activities at molecular level.

\section{MATERIALS AND METHODS}

\subsection{Chemicals and Reagents}

Fetal bovine serum (FBS), Dulbecco's modified eagle medium (DMEM), penicillin G, streptomycin, L-glutamine, phosphatebuffered saline (PBS, $\mathrm{pH}$ 7.4) were purchased from Gibco Life Technologies (GrandIsland, NY, USA). Hygromycin B was purchased from Roche Co. (Basel, Switzerland). Blasticidin S (100 mg) was acquired from Invivogen Asia Co., Ltd (Hong Kong, China). 3-(4,5-Dimethythiazol-2-yl)-2,5 -diphenyltetrazolium bromide (MTT) and tetracycline were obtained from Sigma Chemical Co. (St. Louis, MO, USA). Trizol reagent was acquired from Thermo Fisher Scientific Co., Ltd (Shanghai, China). Synthetic peptides (purity > 95\%) PW5 (PPKNW) and WN5 (WPPKN) were chemically synthesized by GL Biochem (Shanghai) Co., Ltd (Shanghai, China). The peptide was prepared using L-isomers of each amino acid by solid-phase synthesis with the fluorenylmethoxy - cabonyl (Fmoc) method, and was stored at $-20^{\circ} \mathrm{C}$ until use. All other chemicals and reagents used were of analytical grade.

\subsection{Cells}

HEK-293-E22G cells, a cell line constructed by A $\beta 42-$ mCherry (WT) or A $\beta 42(E 22 \mathrm{G})$-mCherry plasmid, were provided by Prof. Alan Tunnacliffe (Department of Chemical Engineering and Biotechnology, University of Cambridge, Cambridge, UK). Cells were cultured in DMEM supplemented with $10 \%$ heat-inactivated FBS containing $100 \mathrm{U} / \mathrm{mL}$ of penicillin, $100 \mu \mathrm{g} / \mathrm{mL}$ of streptomycin, $50 \mu \mathrm{g} / \mathrm{mL}$ of hygromycin $\mathrm{B}, 5 \mu \mathrm{g} / \mathrm{mL}$ of blasticidin $\mathrm{S}$ and $5 \mathrm{mM}$ of L-glutamine at $37^{\circ} \mathrm{C}$ under humidified air with $5 \% \mathrm{CO}_{2}$. Cells in exponential growth phase were used for the experiment.

\subsection{Microscopy}

Cells were routinely propagated in culture medium and treated with vehicle or peptide PW5 or peptide WN5 for $48 \mathrm{~h}$, and then added tetracycline (final concentration: $10 \mu \mathrm{g} / \mathrm{mL}$ ) for $72 \mathrm{~h}$. Subsequently, A $\beta 42$ aggregates in cells were observed under an Olympus IX-73 microscope (200 X).

\subsection{Cell Viability Measurement}

The effects of peptide PW5 and WN5 on the viability of HEK-293E22G cells were determined by MTT assay as mentioned in previous literature [12]. Cells pre-treated with $0.5 \mathrm{mM}$ PW5 and WN5 were routinely propagated in culture medium for $48 \mathrm{~h}$, and then detected the cell viability using MTT method.

\subsection{Cytometers}

Cells $\left(2 \times 10^{5}\right.$ cells/well $)$ were seeded in a 6 -well plate and treated with vehicle or PW5 $(0.5 \mathrm{mM})$ or WN5 $(0.5 \mathrm{mM})$ for $48 \mathrm{~h}$ and then added tetracycline (final concentration: $10 \mu \mathrm{g} / \mathrm{mL}$ ) to induce for $72 \mathrm{~h}$. Then cells were collected and examined by flow cytometer to detect the red fluorescence of $\mathrm{A} \beta$ aggregates in cells. Flow cytometric analysis was performed using FACScan flow cytometer (Beckhan, Sanjose, CA, USA). In order to validate the conventional cytometry results, the $\mathrm{A} \beta$ aggregation in cells was quantified by ImageStream ${ }^{x}$ MKII imaging cytometer. The detection parameters were set up as follows: Channels 01 (bright field) and 04 (fluorescence channels). Magnification was $60 \mathrm{X}$, providing a pixel size of $0.3 \mathrm{um}^{3}$ and the laser $561 \mathrm{~nm}$ activated for fluorescence of mCherry. For simple enumeration of pre-prepared microparticle samples, the acquision cut-off was set to 10000 . The aggregation rate was estimated using formula 1.

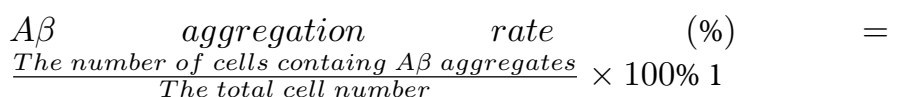

\subsection{RNA-seq Analysis}

RNA-seq was performed on three biological replicates of each group, including Control, Model, PW5 and WN5 group. Cells treated with vehicle, PW5 $(0.5 \mathrm{mM})$ and WN5 $(0.5 \mathrm{mM})$ for $48 \mathrm{~h}$ and added with tetracycline for another $72 \mathrm{~h}$. Collected cells were stored in Trizol reagent at $-80^{\circ} \mathrm{C}$ to further processing. Total RNA was extracted from cell samples and RNA-depleted by using the Ribo-Zero Gold rRNA Remove Kit (Illumina, provided by BGI Company). Sample libraries were made by using TruSeq Constructions Kits and sequenced by using the Hiseq platform (Illumina, provided by BGI Company) with 20-30 million $50 \mathrm{bp}$, paired-end sequences per sample. RNA extraction, rRNA depletion, library construction, and sequencing were done by Covance Genomics Laboratory. Differential gene expression analyses were performed as follows: Fastq reads were aligned to Homo by using Tophat with default settings. HTSeq count was used to get read counts for each sample. Ensemble gene annotation file was used for gene expression quantification. Differential analysis was performed with DESEq. 


\subsection{Proteomics Analysis}

Three separate biological experiments were performed to quantify protein expression. Cells were treated with vehicle, peptides PW5, and WN5 for $48 \mathrm{~h}$, and added with tetracycline for another 72 $\mathrm{h}$. Then, we collected the cells using PBS to obtain the proteins for detection. The further procedures including protein digestion, iTRAQ labeling, strong cation exchange (SCX) fractionation and LC-MS/MS analysis were conducted by BGI Company according to previous literatures $[16,17]$.The proteins in the Model group (vehicle) were labeled with reagent $126,127 \mathrm{~N}$ and $127 \mathrm{C}$, whereas those in the PW5-treated group were labeled with reagent $128 \mathrm{~N}$, $128 \mathrm{C}$, and $129 \mathrm{~N}$, and the WN5-treated group were labeled with reagent $129 \mathrm{C}, 130 \mathrm{~N}$ and $130 \mathrm{C}$. After LC-MS/MS analysis, the raw files were analyzed by using Proteome Discover v.1.4 software. Differential proteins were further analyzed for significant up- or downregulation. The values of the intensities of the three reporter ions for each experimental group were averaged and then the difference was statistically calculated. The fold change was set to $>1.2$ for protein upregulation and $<-1.2$ for protein downregulation. And these proteins were linked to the following databases for further analysis: Gene Ontology (GO) and Kyoto Encyclopedia of Genes and Genomes (KEGG).

\subsection{Statistical Analysis}

All data from experiments were presented as mean \pm SEM (standard error of the mean). Differences between two groups were determined using two-tailed, unpaired Student t-test with Welch's correction. Statistical significance among $>2$ groups with only one variable was assessed using ANOVA followed by Bonferroni's post hoc test. Differences were considered significant at $\mathrm{p}<0.05$.

\section{RESULTS AND DISCUSSION}

\subsection{Measurement of Typical Signatures of the Constructed A $\beta 42$ Aggregation}

Previous study reported that an arctic mutant in $\mathrm{A} \beta 42$ sequence [9], Glu22 (E) to Gly22 (G), could rapidly recruit expressed protein into aggregates inside cells as shown in Figure 1A. Wild-type A $\beta 42$ sequence plasmid was also transfected into cells to further confirm the results of its arctic mutant displayed aggressive aggregation in cells (Figure 1B). Here, to observe the intracellular A $\beta 42$ aggregation (red fluorescence) after tetracycline, microscopy and Amnis imaging flow cytometers (IFCs) were applied to detect the aggregates and fluorescence intensity. The images from Figure 1C illustrated that red fluorescence dots significantly increased in the Model group (mCherry- $\mathrm{A} \beta 42(\mathrm{E} 22 \mathrm{G})$ ), while no aggregates in the Control group (mCherry-A $\beta 42(\mathrm{WT})$ ). In compared with the Control group, the $\mathrm{A} \beta$ aggregation ratio for Model group was calculated to be $77.96 \pm 1.55 \%$ (Figure 1D), and the fluorescence intensity ratio of the mCherry fusion was also dramatically increased (91.6 $\pm 0.21 \%$, Figure 1E) accordingly. Thus, in addition to the consistence to the reported literatures $[9,12,14]$, we provided here high-throughput detection methods for the screening of anti-aggregation compounds through fluorescence measurement of imaging equipment and cytometers.

\subsection{Effect of Arctic Mutant $A \beta 42$ peptide on Transcriptomics in A $\beta 42$ Aggregation Cell Model}

Generally, omics approaches rely on known differences between samples (e.g. treated versus untreated, healthy versus diseased), these seem to be the ideal methods to detect molecular differences (including the expression of genes and the content of proteins) in samples $[16,17]$. Thus, we investigate how the arctic mutant of $A \beta 42$ peptide affects transcriptomics in HEK-293 cells by comparing the mutation and without mutation cells. $\mathrm{A} \beta 42$ arctic mutant has been reported to can generate protofibrils at much lower concentrations and higher rates than without arctic mutant (WT $\mathrm{A} \beta 42)$ in vitro [14]. Transfection with $\mathrm{A} \beta 42$ Arctic mutant plasmid into a stable line cell gene could lead a much faster rate of protein aggregation than WT A $\beta 42$ in living cells, suggesting enhanced protein production or inhibited protein degradation. Messenger RNA is a molecule made when a gene is involved in making its protein. Thus, it is meaningful to explore the alterations of RNA after transfected with $\mathrm{A} \beta 42$ arctic mutant plasmid to form a stable cell model. A comparative transcriptomic analysis was undertaken to explore the molecular mechanism of cell in response to transfection.

In general, the correlation of gene expression level among samples is a key criterion to test whether the experiments are reliable and whether the samples chosen are reasonable. Correlation heatmap was used to display common genes between each two samples based on normalized expression results as Figure 2A. We found that the correlation coefficient values of samples in the Control group were quite different from the Model group, whose correlation value between within group is very close to 1 (violet), implying that biological replicates were well meted and the transfection with arctic mutant $\mathrm{A} \beta 42$ resulted in the alterations of common genes. Furthermore, the principal component analysis (PCA) result (Figure 2B) showed that there was an obvious visual effect of cluster groups between the Control and Model samples, in line with correlation coefficient result. Next, we used NOISeq method to find differential expressed genes (DEGs) between groups, and then 345 genes (233 up-regulated, 112 down-regulated) were identified as DEGs caused by the arctic mutant as shown by the Volcano graph Figure 2C. Because of genes with similar expression patterns usually have same functional correlation, so we further perform clustering analysis of DEGs with cluster. The result from Figure 2D showed that two cluster represented the DEGs between the Control and Model group. What's more, fold changes of expression between DEGs were also presented as heatmap Figure 2D, indicated that Arctic mutant markedly changed the transcripts.

Subsequently, according to gene ontology (GO) functional analysis result, we found that DEGs in the Model group were mainly involved the cell, cell part, membrane, and organelle. In transcript level, arctic mutant resulted in the abnormal aggregates might through regulating the molecular and biological functions in the binding, catalytic activity, cellular process, metabolic process, biological regulation, and multicellular organismal process and so on. Furthermore, GO classification analysis was applied to help understand the distribution of gene functions of the specie from the 
Figure 1

A

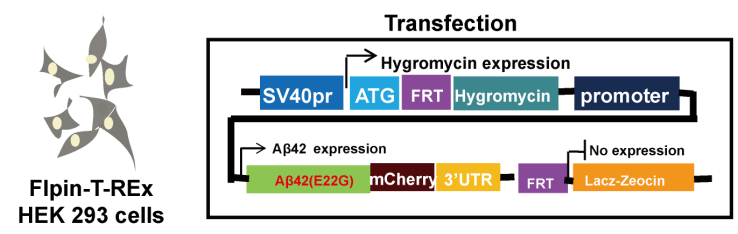

C

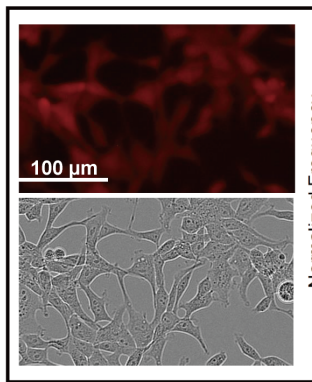

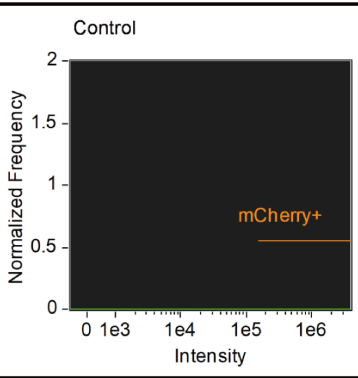

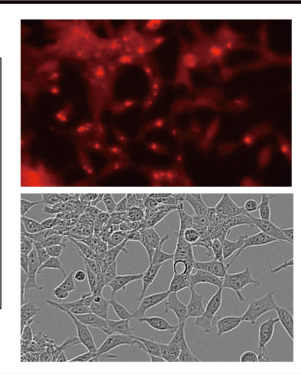

B

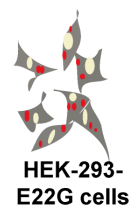

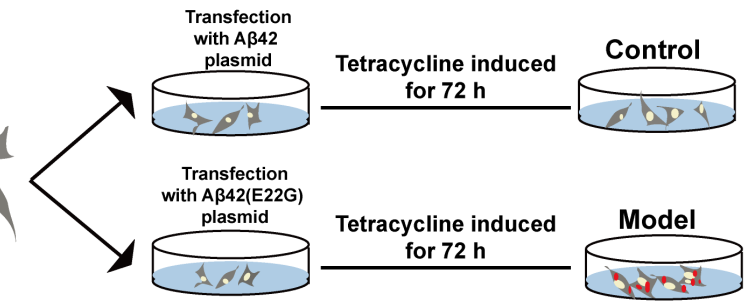

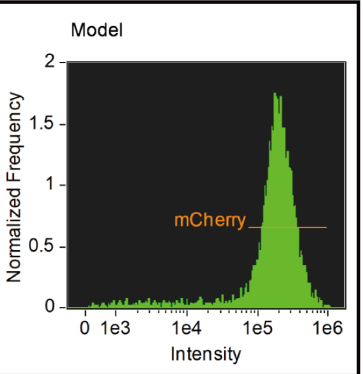

D

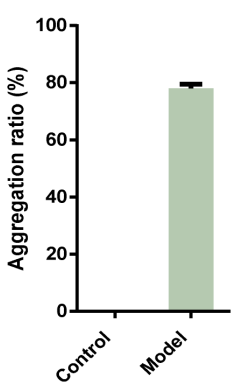

E

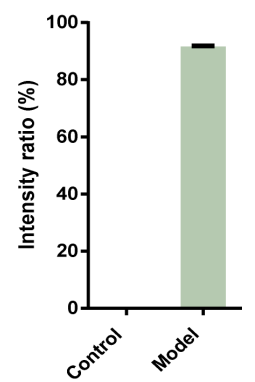

Figure 1 A $\beta 42$ arctic mutant in cell model displays a fast and aggressive aggregation phenotype. (A) Schematic representation of A $\beta 42$ protein fast aggregation inside cells. (B) Schematic representation of mCherry-A $\beta 42$ (WT) and mCherry-A $\beta 42$ (E22G) aggregation. (C-E) Images and quantification results showed that $\mathrm{A} \beta 42$ arctic mutant in cell model leads a fast and aggressive aggregation. The data are representative of three independent experiments.

macro level using WEGO software. As shown in Figure 2E, for arctic mutant intervention, DEGs were mostly enriched for five profiles, including negative regulation of myeloid cell differential, negative regulation of cellular component movement, extracellular matrix organization, morphogenesis of a branching structure, and negative regulation of locomotion. Proteins must be folded into their final form in order to function normal, otherwise protein misfolding will lead to the occurrence of diseases, such as AD and Parkinson's disease (PD) [18]. The brain tissue of AD patients is full of misfolded proteins like $\mathrm{A} \beta$ plaques [19]. And the mechanisms implicated in the formation large aggregates of these misfolded proteins are related to molecular movements, folding, and assembling [18, 20], which are consistent with the above results of five DEGs enriched profiles in the Model group. Kyoto Encyclopedia of Genes and Genomes (KEGG) pathway analysis showed that DEGs enriched in the signal transduction, signaling molecules and interaction, neurodegenerative diseases, immune diseases, lipid metabolism, etc., which are closely associated with the development of AD. This data was consistent with previous studies in the transcript level of $\mathrm{AD}$ [21]. Therefore, the cell model constructed by the arctic mutant of the $\mathrm{A} \beta 42$ sequence plasmid not only could use for quickly high-throughput screening the potential substances in vitro, but also provides significant insights into finding the potential AD therapeutic targets at the molecular level that might be beneficial for further in vivo tests.

\subsection{Effects of Walnut-derived Peptides PW5 and WN5 on the Anti-A $\beta 42$ Aggregation Activity}

In the present work, we compared peptide PW5 (Pro-Pro-Lys-AsnTrp, 640.5 Da) with another walnut-derived peptide, WN5 (TrpLys- Pro-Pro-Asn, $640.5 \mathrm{Da})$, in anti-A $\beta 42$ aggregation activity using the HEK-293-E22G cell model. Although the amino acids composition of PW5 and WN5 peptides was the same, the sequence was different. Interestingly, we found that two peptide all possessed good anti-A $\beta 42$ aggregation activity, but there existed slightly different efficacy between them. Firstly, the red dots of A $\beta 42$ aggregates were significantly reduced by peptide PW5 and WN5 as shown by Figure 3A.

To rule out the possibility that cell number or toxicity could have affected the results, we measured cell viability using MTT assay and cell counting. We found that the concentration of PW5 and WN5 at $500 \mu \mathrm{g} / \mathrm{mL}$ had no obvious toxicity and proliferation effects on HEK-293-E22G cells ( $\mathrm{p}>0.05$, Figure 3B), implying that PW5 and WN5 could effectively inhibit the intracellular $\mathrm{A} \beta$ aggregates in vitro. Further, to verify the anti-aggregation activity of these two peptides in vitro, flow cytometry and Amnis imaging flow cytometers were applied. In comparison with the Model group, both PW5 and WN5 could obviously decrease the intracellular $\mathrm{A} \beta$ aggregates, including the aggregation ratio and fluorescence intensity, respectively ( $\mathrm{p}<0.05$, Figure $3 \mathrm{C}-\mathrm{E}$ ), although PW5 slightly better than that of WN5. In addition, the percentage of aggregation dot in every collected cell was auto-recorded and calculated by IFCs, whose data might be more accurate for analysis. As shown in the Figure 3F, the percentage of no $\mathrm{A} \beta$ aggregation dot inside cell in the PW5 $(66.03 \pm 0.89 \%)$ and WN5 (62.9 \pm 
Figure 2

A

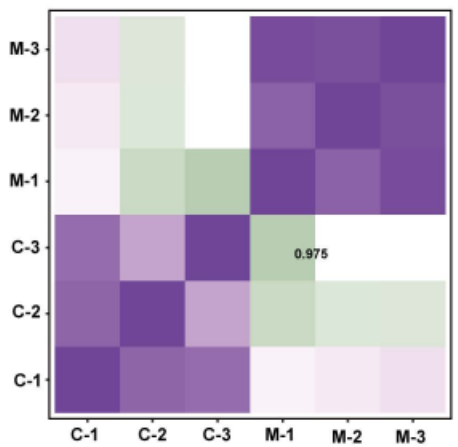

B

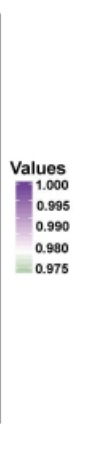

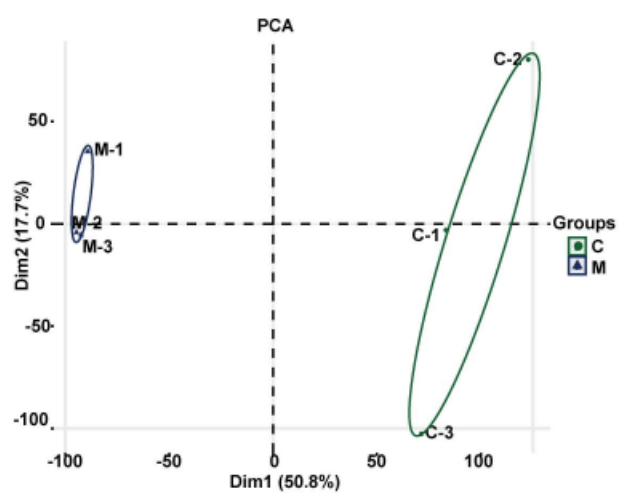

C

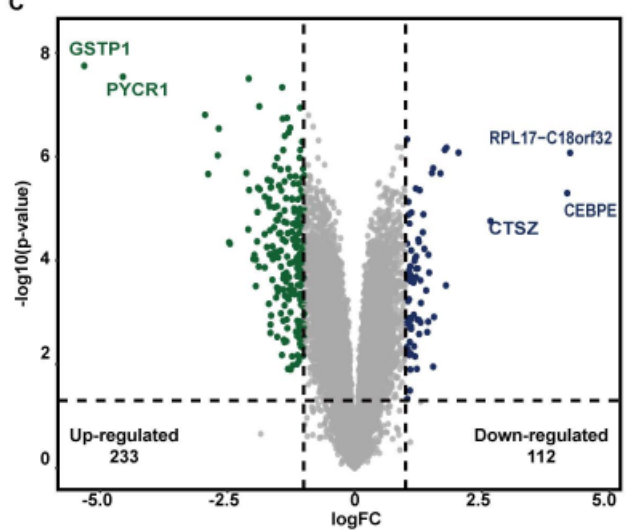

D

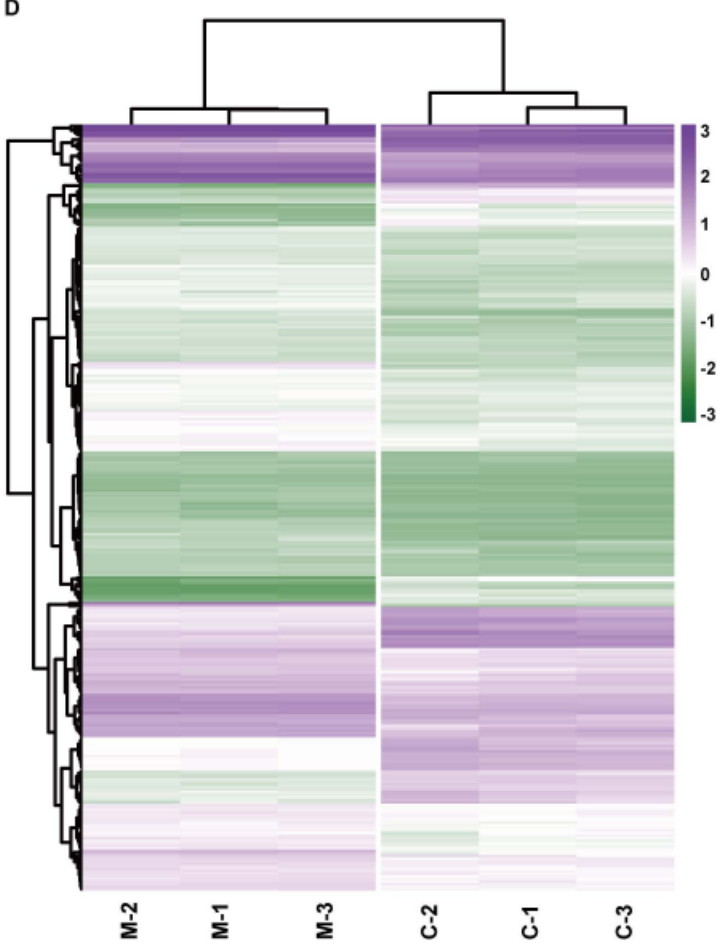

E

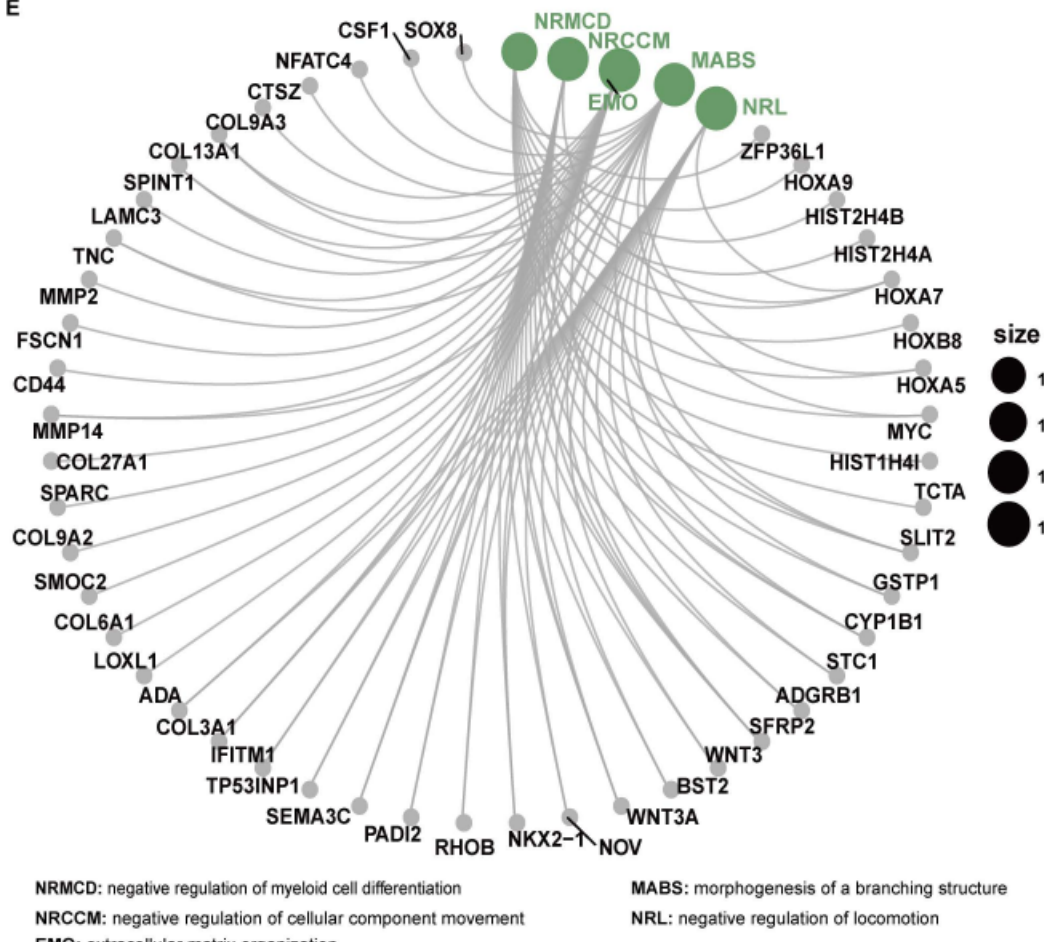
NRCCM: negative regulation of cellular component movemen NRL: negative regulation of locomotion EMO: extracellular matrix organization

Figure 2 Effectof Arctic mutant $\mathrm{A} \beta 42$ peptide on transcriptomics in $\mathrm{A} \beta 42$ aggregation cell model. (A) Heat map of correlation coefficient values acrossing the mCherry-A $\beta 42(\mathrm{WT})(\mathrm{C}, \mathrm{C}-1, \mathrm{C}-2$, and $\mathrm{C}-3)$ and mCherry-A $\beta 42(\mathrm{E} 22 \mathrm{G})(\mathrm{M}, \mathrm{M}-1, \mathrm{M}-2$, and M-3) aggregation groups. (B)Samples could be clustered according to the value distribution of component 1 and component 2 in the $\mathrm{C}$ and $\mathrm{M}$ groups using the Principle component analysis (PCA). (C)Volcano graph of all expressed genes in each pairwise (C vs M). Dots in green mean significant differential expressed genes (DEGs) which passed screening threshold and deep blue dots are non-significant DEGs. (D) The union heat maps showed the hierarchical clustering of DEGs for each sample from C and M groups. (E) Go functional classification on DEGs in each pairwise (C group vs M group). 
Figure 3

A
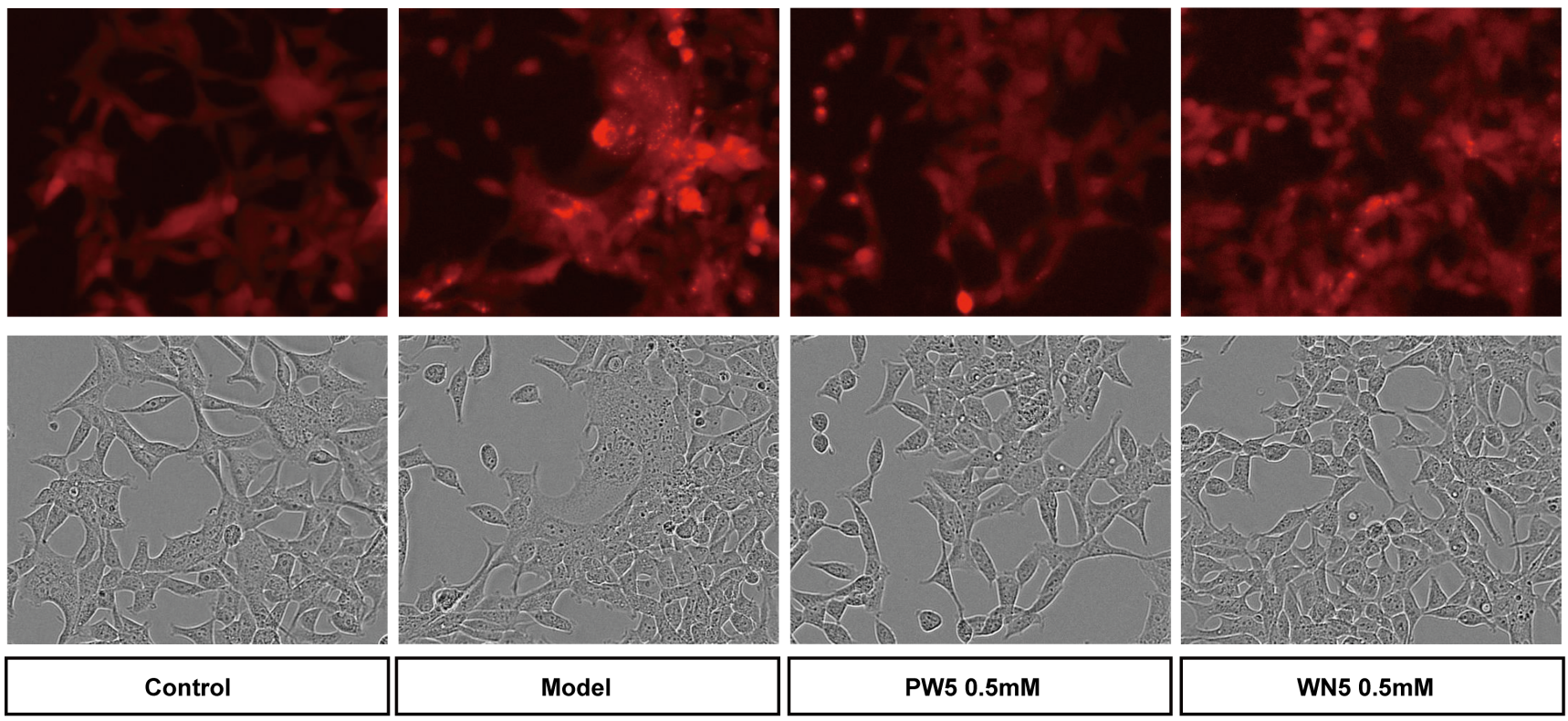

B
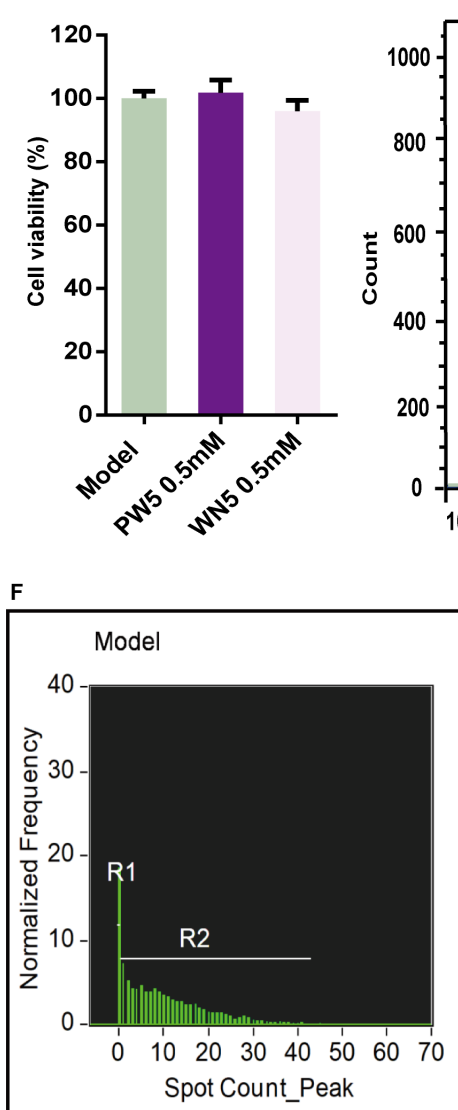

C

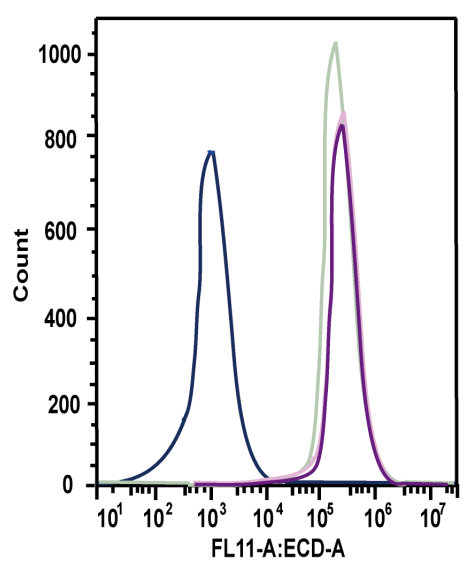

FL11-A:ECD-A
D

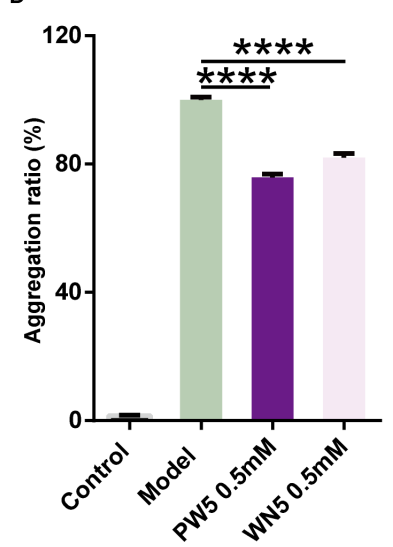

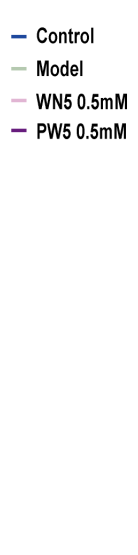

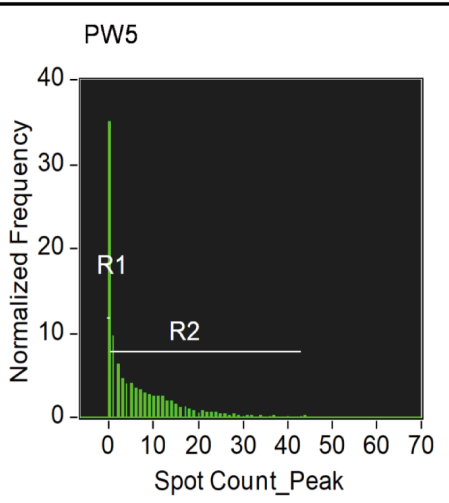

E
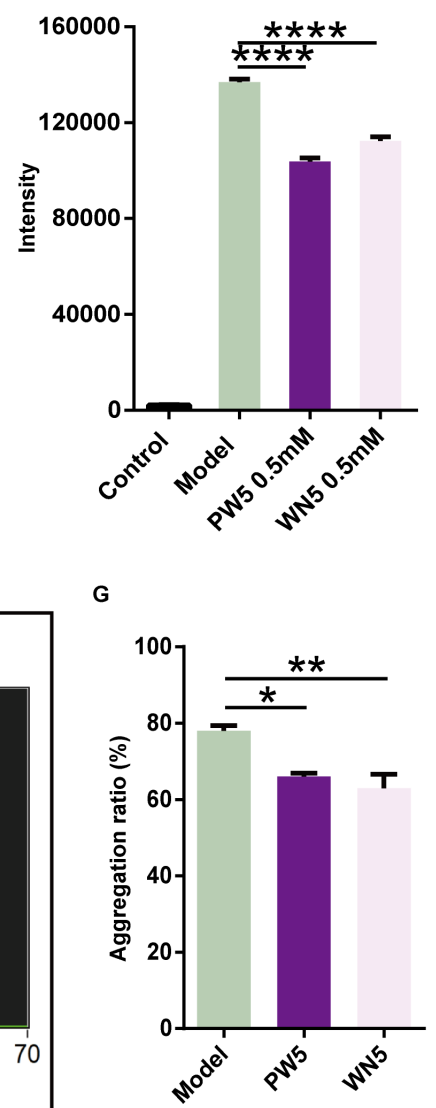

Figure 3 Anti-aggregates activity of walnut-derived peptides PW5 and WN5 in vitro. (A) Images of $\beta 42$-aggregates inHEK-293-E22G cells after treated with vehicle, PW5, and WN5, using an IncuCyte Zoom live cell imaging system. From left to right, Control group (cells without A 342 -aggregates), Model group, PW5 group $(0.5 \mathrm{~mm})$, and WN5 group (0.5 mM). (B) Results of cell viability after treated with PW5 and WN5 at the concentration of $0.5 \mathrm{mM}$. (C) Histogram results of anti-A $\beta 42$ aggregation activity of PW5 and WN5 by cytometry. (D)Results of anti-A $\beta 42$ aggregates ratio in cells after treated with PW5 or WN5. (E) Results of A $\beta 42$ aggregates intensity in cells after treated with PW5 orWN5. (F)The mCherry fluorescence of $\mathrm{A} \beta 42$ aggregates results. $(\mathrm{G}) \mathrm{A} \beta 42$ aggregation ratio results. Statistical analysis was performed by ANOVA $\left({ }^{*} \mathrm{p}<0.05\right)$. The data are presented as mean \pm SEM. 
$3.71 \%)$ groups were markedly higher than the Model group (77.97 $\pm 1.38 \%, \mathrm{p}<0.05)$. And then the $\mathrm{A} \beta$ aggregation ratio was calculated according to the results of the percentage of no $\mathrm{A} \beta$ aggregation dot inside cells. The result (Figure 3G) showed that PW5 or WN5 intervention resulted in reduced $\mathrm{A} \beta$ aggregation $(\mathrm{p}<$ 0.05 ), especially WN5. These results illustrated that walnut-derived peptides could effectively inhibit $\mathrm{A} \beta$ aggregation over time in cells, and provide some critical information for them further applied in functional foods or other fields.

Furthermore, we found a few interesting points that different effects on anti-aggregation activity were varied from PW5 and WN5. It is thought that various kinds of biological activities depended on the difference in molecular weight, amino acid sequence, amino acid composition and conformation of peptide [22, 23]. Different amino acids sequences are related to the different side chains of amino acid, and also the groups of side chains make important sense to the diversities of peptides functions. Zheng et al. found that Tyr- and Trp-containing dipeptides with Tyr/Trp residue at the N-terminus showed stronger antioxidant activities than that at the C-terminus, and the neighboring residue also affected their activities by steric effect, hydrophobicity and hydrogen bonding among others [24]. Thus, this different result from PW5 and WN5 might be associated with their own amino acids sequences but not amino acids composition and molecular weight. More importantly, the underlying different possible reasons also could be further investigated in the transcript and translation level.

\subsection{Effects of Peptide PW5 or WN5 on Transcriptomics in A $\beta 42$ Aggregation Cell Model}

Prior to DEG analysis, the correlation coefficient value among samples was examined for various treatments. Correlation coefficient value distribution in heatmap (Figure S1, Appendix A) showed that most of the genes belonged to the same group leading to three clusters, especially between the Model group and PW5 or WN5 groups. The Cluster analysis showed (Figure 4A) that the genes expression between samples distribution in heatmap was not obvious, implying that little transcript changes occurred after walnut-derived peptides treatments. However, the PCA analysis demonstrated that the distance of relationship between samples was still apparent far from each other along the component 1 (29.1\%) and component $2(19.3 \%)$ axes (Figure 4B), signified that some factors of sample variation were still important for treated groups in comparison with Model group. To further explore this difference in gene expression, DEGs were screened according to the expression difference multiple $\mid \log 2$ Fold-change $\mid>1$ and significant $\mathrm{p}<0.05$. Surprisingly, the PW5 treatment group only contained 5 significantly upregulated DEGs and 1 significantly downregulated DEGs. Also the WN5 group contained 7 significantly upregulated DEGs and 7 significantly downregulated DEGs, that slightly more than PW5 group (Figure S2, Appendix A). Clustering analysis showed that DEGs between Model and PW5 group were divided into two groups with variation of four upregulated DEGs in PW5 group (Figure 4C), and the DEGs between Model and WN5 group were also divided into two groups with variation of 6 upregulated DEGs and 3 downregulated DEGs in WN5 group (Figure 4D).
Gene annotation analysis showed that the differential expression genes had encoded binding, cellular process and cell part after PW5 intervention, as well as the DEGs in WN5 group encoding binding, cellular process, metabolic process, and cell part, etc (Figure S3. Appendix A). Moreover, analysis of enriched KEGG pathway for DEGs revealed that these transcripts were assigned to multiple reference pathways, especially the Ribosome. Because of there were just a few DEGs both in the PW5 and WN5 groups, then the results of GO and KEGG pathways analysis were unsupportive for drawn the final conclusion. In general, PW5 and WN5 treatments could effectively decrease $\mathrm{A} \beta 42$ aggregates, while have little effects on transcript level in Arctic mutant cell model. This also suggests an important point that walnut (food)-derived peptides may have fewer side effects due to little effects on transcription level and then show huge potential advantages in the application of functional food or drugs.

\subsection{Effects of Peptide PW5 or WN5 on Proteomics in A $\beta 42$ Aggregation Cell Model}

Gene is the guider of life activities, and protein is the final executor, so as to the transcriptome analysis can only provide us with onesided information at the transcription level of the life activities [5]. Thus, proteomic analysis in translational level could be very helpful understanding changes in response to peptides treatments in this arctic mutant cell model. In the present study, clustering analysis showed (Figure 5A) that differential expressed proteins between PW5/Model and WN5/Model groups were divided into two groups, suggested that different peptide treatment caused differing results in translation level. On the basis of our analysis, we identified 5784 proteins from a total of 28719 peptides. There was a significant difference in the expression of proteins between the Model and PW5 treated groups, among which 98 were upregulated and 86 were downregulated after PW5 intervention (Table S1, Appendix A). We also found that 194 differential proteins between the Model and WN5 treated groups, among these, 111 were upregulated and 83 downregulated in WN5 treated group (Table S1, Appendix A). This indicated that larger changes of the translation level in the WN5-treated group were measured compared to PW5 intervention. Furthermore, by comparison with PW5 group, WN5 group with a significant difference in 54 between the PW5 and WN5 groups. Among these, 28 were upregulated and 26 were downregulated in the WN5 group (Table S2, Appendix A), implying that although not much of a change existed between the two groups in translation level, the changes might contribute to the difference in their antiA $\beta 42$ aggregation activity.

Furthermore, according to KEGG pathway enrichment analysis showed that there were 15 and 9 statistically significant pathways in PW5/Model and WN5/Model groups, respectively (Table S3, Appendix A). Moreover, one common pathway, whose function in mismatch repair, was shared within two groups. There are several DNA repair mechanisms in the human body, and the base excision repair (BER) is reported that plays a crucial role in degenerative diseases, including AD [25]. Although differential proteins enriched in mismatch repair pathway were slightly different between the PW5 (MSH2, MSH3, RFA3, and DPOD1) and WN5 (MSH2, MSH6, RFA3, and RFA1) group, indicated that this pathway plays a critical 
Figure 4

A

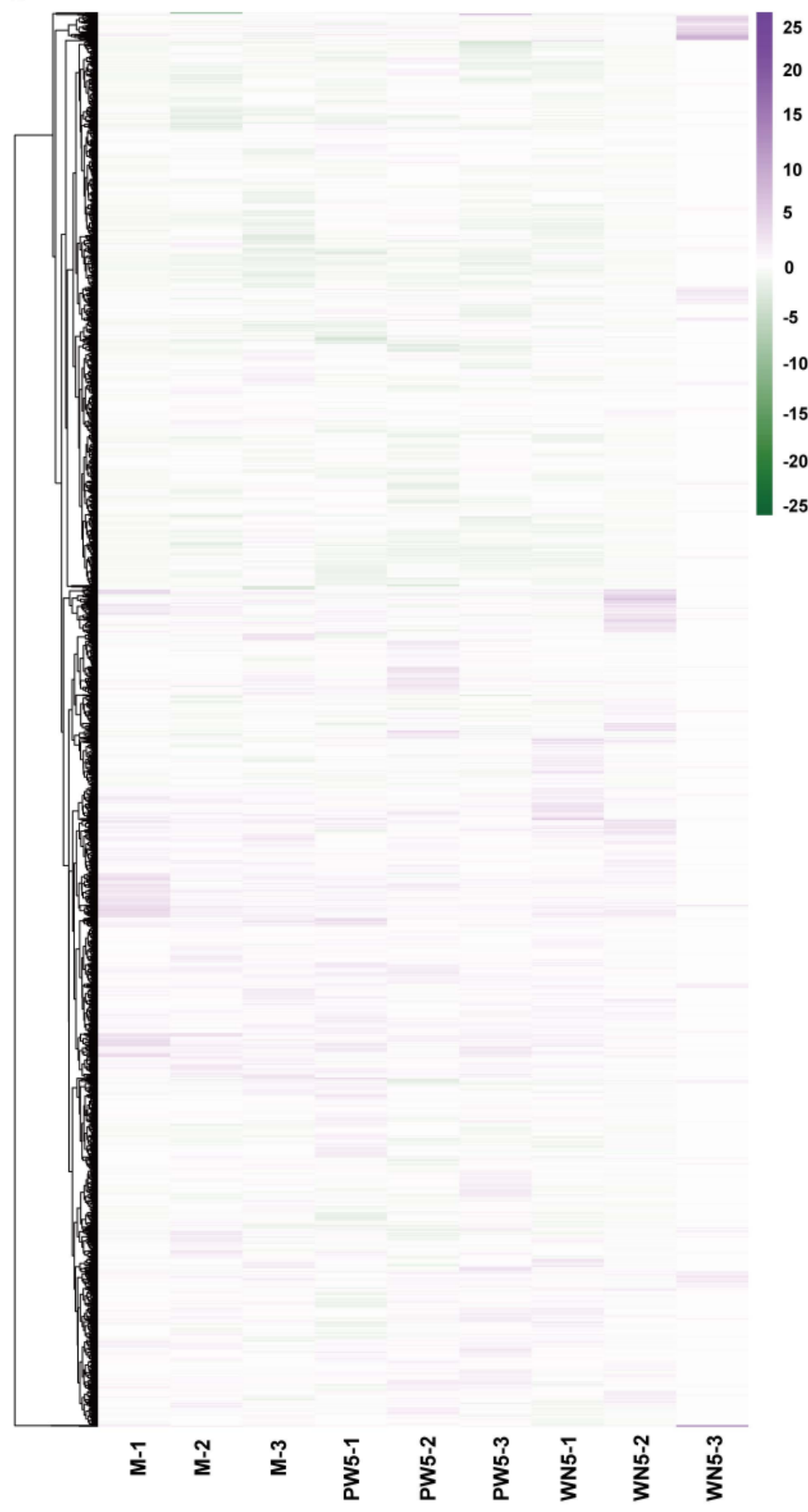

B

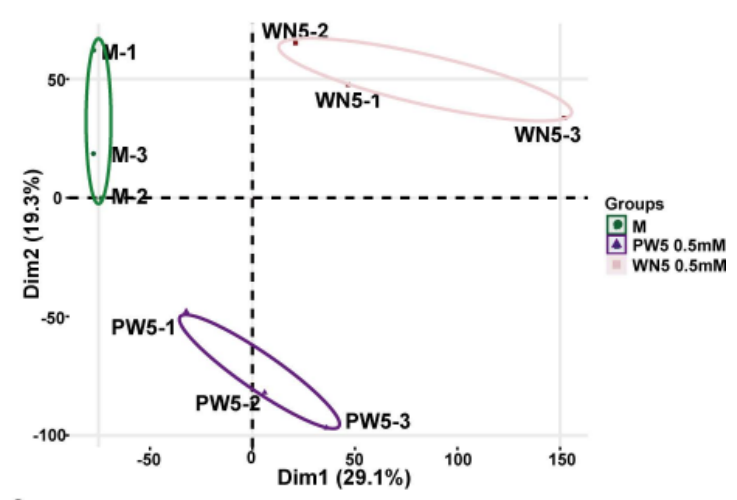

C

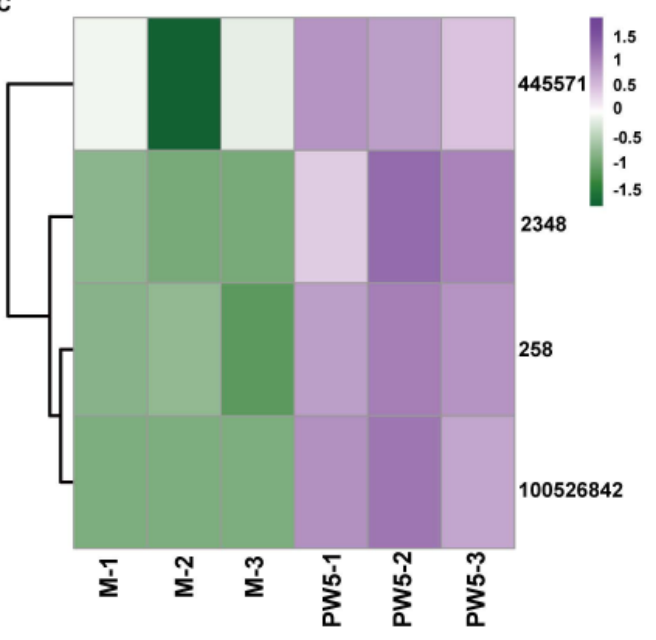

D

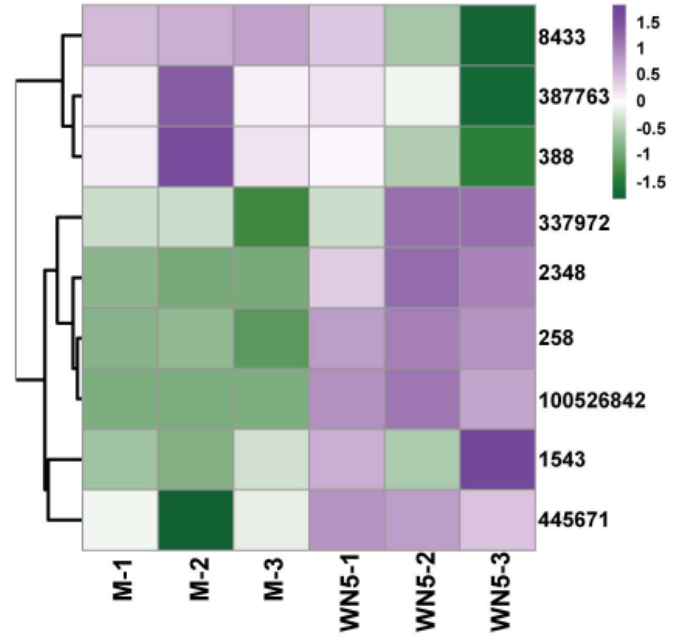

Figure 4 Effects of peptide PW5 or WN5 on transcriptomics in A $\beta 42$ aggregation cell model. (A) Heatmap showed the hierarchical clustering of DEGs in the Model group (M-1, M-2, and M-3), PW5 group (PW5-1, PW5-2, and PW5-3) andWN5 group (WN-5, WN5-2, and WN5-3). (B) Samples could be clustered according to the value distribution of component 1 and component 2 from Model, PW5 and WN5 groups using PCA analysis. (C) Heatmap sanalysis of Model and PW5 treated group. Gene name: 445571: COBW domain containing 3; 2348: folate receptor alpha 1; 258: ameloblastin; 100526842: RPL17-C18orf32. (D) Heatmaps analysis of Model and WN5 treated group. Gene name: 8433: undifferentiated embryonic cell transcription factor 1;387763: chromosome 11 open reading frame 96; 388: ras homolog family member B; 337972: keratin associated protein 19-5; 2348: folate receptor alpha 1; 258: ameloblastin; 1543: cytochrome P450 family 1 subfamily Amember 1; 445671: alcohol dehydrogenase class 3. 
A

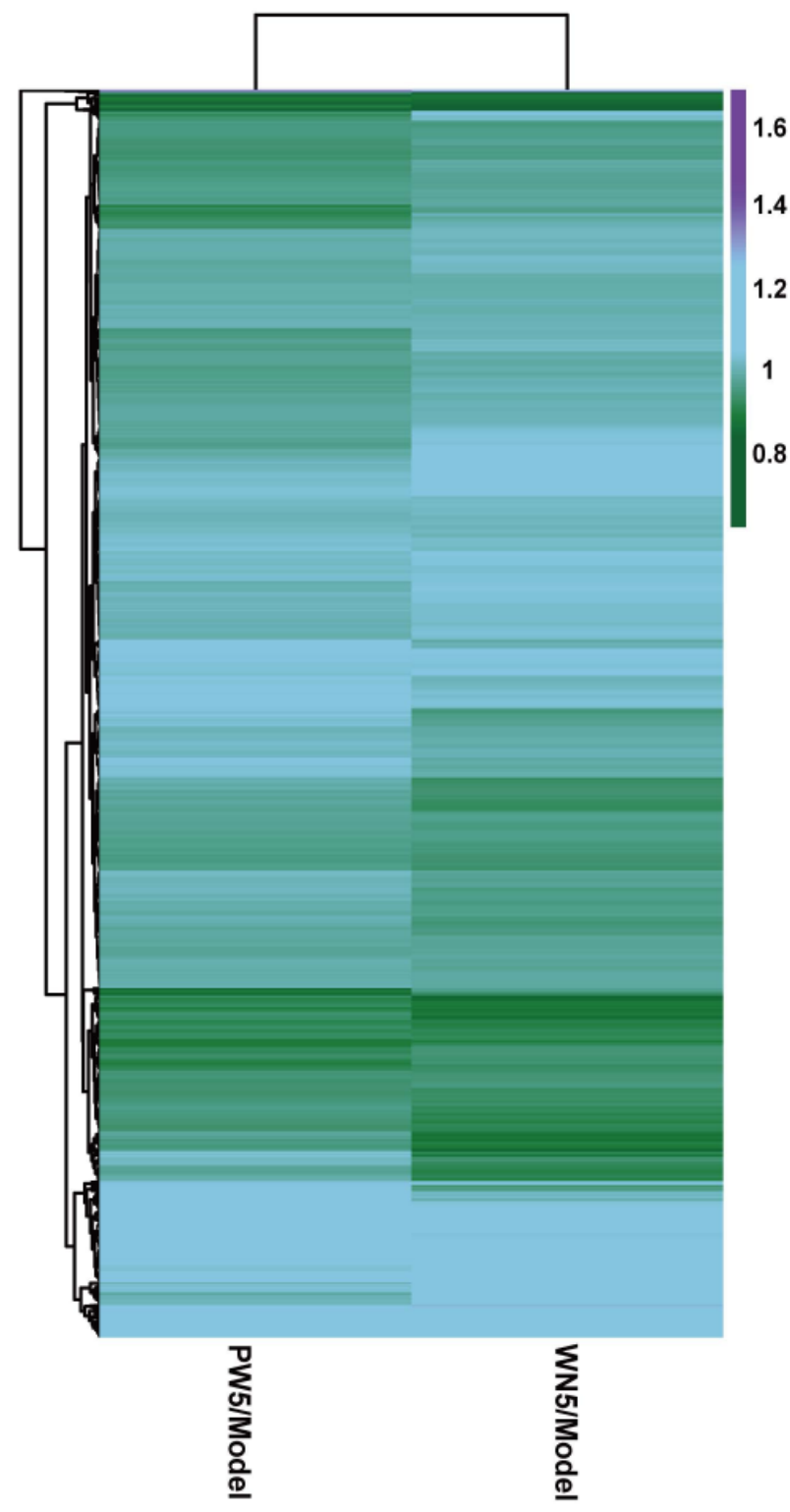

B

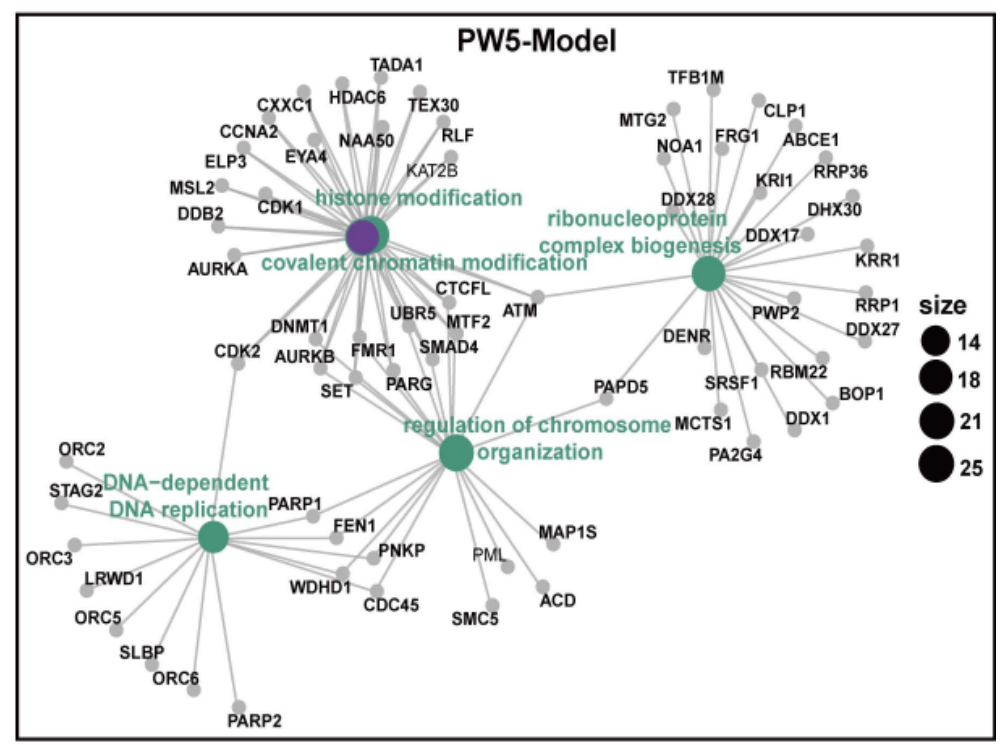

c

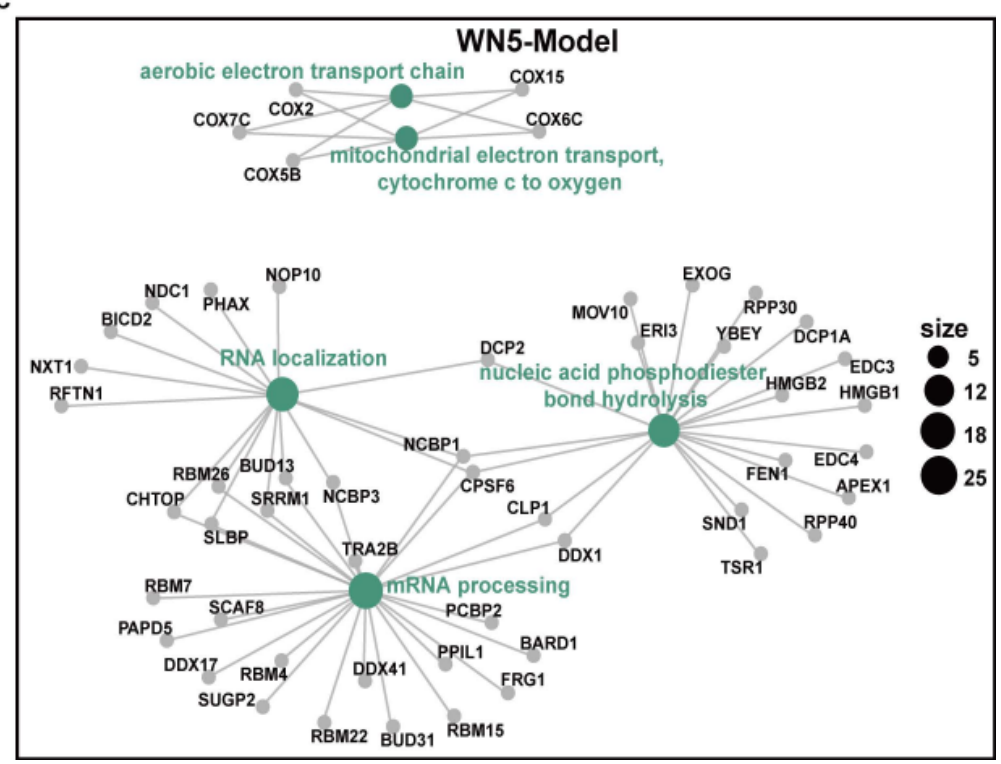

Figure 5 Effects of peptide PW5 or WN5 on proteomics in A $\beta 42$ aggregation cell model. (A) Heatmap showed the hierarchical clustering of proteins in Model group/PW5 group and Model/WN5 group. (B) Network analysis of protein expression for Model group/PW5group. (C) Network analysis of protein expression for Model group/WN5 group.

role in their anti-aggregation activity. In addition, only one KEGG pathway, TGF- $\beta$ signaling pathway (differential proteins such SMAD3 and PITX2), was enriched when WN5 group compared to PW5 group (Figure S4, Appendix A). Vladimir et al. found that one drug could target to TGF- $\beta$ receptor in astrocytes that inhibit inflammation in the brain may slow or even reverse cognitive decline with age diseases like $\mathrm{AD}$ [26]. A bioinformatics analysis study has reported that DEGs in the mild AD group were mainly enriched in the TGF- $\beta$ signaling pathway [27]. These indicated the differential proteins SMAD3 and PITX2 in TGF- $\beta$ signaling pathway might contribute to the better performance of WN5 treatment than PW5.
Proteins often interact to form protein-complexes to perform their functions.According to the protein-protein interaction network analysis, the five major clusters of interaction in PW5/Model group were protein processing in histone modification, covalent chromatin modification, ribonucleoprotein complex biogenesis, regulation of chromosome organization, and DNA-dependent DNA replication (Figure 5B). In recent years, emerging evidence shows that epigenetic biology such as histone modification, DNA methylation, chromatin remodeling, plays a role in $\mathrm{AD}$ [28, 29].RNA-seq and ChIP-seq analysis demonstrated that the majority of long noncoding RNAs (lncRNAs) may be transcriptionally regulated by histone modification in $\mathrm{AD}$ [30]. These studies revealed that there was a relationship between epigenetic factors 
and $\mathrm{AD}$, and further exploration focused on this could be better understand the pathogenesis of $\mathrm{AD}$ and develop new therapeutic targets for AD. So, in this work, we found that PW5 treatment might have an effect on epigenetic modification and this may benefit for $\mathrm{AD}$ treatment. As shown in Figure 5C, there were also five clusters of interaction in WN5/Model group, including aerobic electron transport chain, mitochondrial electron transport cytochrome $\mathrm{c}$ to oxygen, RNA localization, mRNA processing, and nucleic acid phosphodiester bond hydrolysis. It was found that several cyclooxygenase (COX) proteins including COX2, COX5B, COX6C, COX7C and COX15 were interacted to form protein-complexes to anticipate in energy metabolism (e.g. aerobic electron transport chain, mitochondrial electron transport cytochrome c to oxygen) [31]. Previous researches have reported that mitochondria dysfunction in neuron cells could be caused by the $\mathrm{A} \beta$ deposition, especially intracellular $\mathrm{A} \beta 42$ [32]. These suggested that the intracellular $\mathrm{A} \beta 42$ abnormal aggregates cell model we used affected the energy metabolism and WN5 treatment might reverse this dysfunction to reduce protein aggregates inside cells. In addition, RNA directly regulates a large number of cell processes and then the protein-complex in RNA localization and mRNA processing play an important role in maintaining homeostasis.

In general, a single-copy plasmid was consisted of an arctic mutant in A $\beta 42$ sequence, Glu22 (E) to Gly22 (G), with a Cytomegalovirus (CMV) promoter and fluorescence label protein mCherry, and then transfected into stable cell line that leading to rapidly recruit expressed protein into aggregates inside cells. The formation of abnormal aggregates might be produced two ways, including increased production and inhibited degradation. Obviously, the molecular level, such as transcript and translation, after transfection with this Arctic mutant plasmid was changed. After receiving the intervention of walnut-derived pentapeptide PW5 or WN5, that was composed of the same amino acid with different sequences, the intracellular $\mathrm{A} \beta 42$ abnormal aggregates was significantly reduced. Then, how do these peptides achieve the goal of lowering the $\mathrm{A} \beta 42$ protein level inside the cells. As you know, the final expression of proteins is related to the gene encoding, gene transcript and translation processes, and could be affected by them. Recent advances in high-throughput technologies enable quantitative monitoring of the abundance of various biological molecules and their variation between various biological states on a genomic scale [33]. Integrated analysis of transcriptomic and proteomic data can provide crucial insights into the metabolic mechanisms underlying complicated biological systems [34]. However, because the relationship between protein abundance and mRNA expression level is complicated by many cellular and physical processes, sophisticated statistical models need to be developed to capture their relationship [35]. In our study, we found that walnut-derived peptides have little effects on transcript level, only changed several significant difference genes, compared to vehicle-treated group. However, the protein level, namely translation level, was markedly altered by peptides' interventions. Although the data of transcriptome is inconsistent with that of proteome, but this implied that post transcriptional regulation might play a more important role in the process $[4,36]$. Based on these results, we could deduce that walnut peptides function might be through the inhibition of $\mathrm{A} \beta 42$ protein production or enhancement the degradation in post transcriptional regulation or translation level.

\section{CONCLUSION}

In this work, our results present the first transcriptomic and proteomic studies to detect the alterations at the molecular level in the Arctic mutant A $\beta 42$ (Glu22 (E) to Gly22 (G) in A $\beta 42$ sequence) cell model with abnormal intracellular aggregates. Owing to the omics data, we not only were able to find the differentially expressed genes or proteins related to the formation of abnormal protein aggregation in the progression of $\mathrm{AD}$, but also provided reference molecular mechanism for screening antiA $\beta 42$ aggregation substances for AD treatment. More importantly, these valuable empirical data that might support future molecular research on food-derived substances in the application of functional foods or AD-related diseases potential drugs.

\section{FUNDING}

The study was funded by the fellowship of China Postdoctoral Science Foundation (No. 2020M672644).

\section{ABBREVIATIONS}

A $\beta$, $\beta$-amyloid; AD, Alzheimer's disease; WW4, Trp-Asp-Gln-Trp PW5, Pro-Pro-Lys-Asn-Trp; WN5, Trp-Pro-Pro-Lys-Asn; WE7, Trp-Ser-Arg-Glu-Glu-Gln-Glu APP, $\beta$-amyloid precursor protein; PD, Parkinson's disease; PCA, principle component analysis; GO, gene ontology; KEGG, Kyoto encyclopedia of genes and genomes; SEM, standard error of the mean; DEGs, differential expressed genes; BER, base excision repair; COX, cyclooxygenase.

\section{CONFLICTS OF INTEREST}

The authors declare that they have no competing interests.

\section{AUTHORS' CONTRIBUTION}

MW designed the study, performed the experiments and interpreted the findings. CCG provided analysis for the study. KMA wrote the manuscript. JYR designed and supervised the study. All authors read and approved the final manuscript.

\section{ACKNOWLEDGEMENTS}

We would thank Prof. Alan Tunnacliffe (Department of Chemical Engineering and Biotechnology, University of Cambridge, Cambridge, UK) for providing the constructed mCherry-A $\beta 42$ and mCherry-A $\beta 42(\mathrm{E} 22 \mathrm{G}) \mathrm{HEK}-293$ cell lines.

\section{A. SUPLEMENTARY INFORMATION}

Supplementary data to this article can be found online at https://do i.org/10.53365/efood.k/144885.

Figure S1 Correlation coefficient value distribution in heatmap of Model, PW5 and WN5 groups. 
Figure S2 Differential expression genes of upregulated and downregulated in PW5/Model and WN5/Model groups.

Figure S3 Gene annotation analysis in PW5/Model and WN5/Model groups.

Figure S4 KEGG pathway enrichment analysis between WN5 and PW5 group.

Table S1 Differential proteins of upregulated and downregulated in PW5/Model and WN5/Model groups.

Table S2 Differential proteins of upregulated and downregulated when PW5 compared to WN5 group.

Table S3 tatistically significant KEGG pathways in PW5/Model and WN5/Model groups.

\section{REFERENCES}

[1] He J, Xia CL, He YX, Pan DD, Cao JX, Sun YY. Proteomic responses to oxidative damage in meat from ducks exposed to heat stress. Food Chem. 2019;295:127-135.

[2] Khan TA, Yusuf M, Ahmad A, Bashir Z, Saeed T, Fariduddin Q. Proteomic and physiological assessment of stress sensitive and tolerant variety of tomato treated with brassinosteroids and hydrogen peroxide under low-temperature stress. Food Chem. 2019;289:500-511.

[3] Schwanhausser B, Busse D, Li N, Dittmar G, Schuchhardt J, Wolf J. Global quantifcation of mammalian gene expression control. Nature. 2011;473(7347):337-342.

[4] Hanada T, Weitzer S, Mair B, Bernreuther C, Wainger BJ, Ichida J. CLP1 links tRNA metabolism to progressive motor-neuron loss. Nature. 2013;495:474-480.

[5] Haider S, Pal R. Integrated analysis of transcriptomic and proteomic data. Curr Genomics. 2013;14(2):91-110.

[6] Golde TE. The A $\beta$ hypothesis: leading us to rationally-designed therapeutic strategies for the treatment or prevention of Alzheimer disease. Brain Pathol. 2005;15(1):84-87.

[7] Guo Q, Li H, Cole A, Hur J, Li Y, Zheng H. Modeling Alzheimer's disease in mouse without mutant protein overexpression: cooperative and independent effects of $\mathrm{A} \beta$ and tau. PloS one. 2013;8(11):e80706-e80706.

[8] Nilsberth C, Westlind-Danielsson A, Eckman CB, Condron MM, Axelman K, Forsell C. The "Arctic" APP mutation (E693G) causes Alzheimer's disease by enhanced $\mathrm{A} \beta 42$ protofibril formation. Nat Neurosci. 2001;4(9):887-893.

[9] Lu M, Williamson N, Mishra A, Michel CH, Kaminski CF, Tunnacliffe A. Structural progression of amyloid- $\beta$ arctic mutant aggregation in cells revealed by multi-parametric imaging. J Biol Chem. 2019;294(5):1478-1487.

[10] Power R, Prado-Cabrero A, Mulcahy R, Howard A, Nolan JM. The role of nutrition for the aging population: implications for cognition and Alzheimer's disease. Annu Rev Food Sci Technol. 2019;10:619-639.

[11] Poulose SM, Miller MG, Shukitt-Hale B. Role of walnuts in maintaining brain health with age. J Nutr. 2014;144(4):561S-566S.

[12] Wang M, Amakye WK, Guo L, Gong C, Zhao Y, Yao MJ. Walnutderived peptide PW5 ameliorates cognitive impairments and alters gut microbiota in APP/PS1 transgenic mice. Mol Nutr Food Res. 2019;63:1900326-1900326.

[13] Chen N, Yang H, Sun Y, Niu J, Liu S. Purification and identification of antioxidant peptides from walnut (Juglans regia L.) protein hydrolysates. Peptides. 2012;38(2):344-349.
[14] Yang L, Lin X, Li Q, Wang M, Zhou M, Wang W. Identification of two novel peptides with antioxidant activity and their potential in inhibiting amyloid- $\beta$ aggregation in vitro. Food Funct. 2019;10:1191-1202.

[15] Wang M, Sun X, Luo W, Božović S, Gong CC, Ren JY. Characterization and analysis of antioxidant activity of walnutderived pentapeptide PW5 via nuclear magnetic resonance spectroscopy. Food Chem. 2020;339:128047-128047.

[16] Li YD, Li X, Xu WB, Han ZB, Zhao YY, Dong J. Comparative iTRAQ-based quantitative proteomic analysis of the Chinese grass shrimp (Palaemonetes sinensis) infected with the isopod parasite Tachaea chinensis. Parasit Vectors. 2019;12:415-415.

[17] Savitski MM, Zinn N, Faelth-Savitski M, Poeckel D, Gade S, Becher I. Multiplexed proteome dynamics profiling reveals mechanisms controlling protein homeostasis. Cell. 2018;173:260274.

[18] Ciechanover A, Kwon YT. Degradation of misfolded proteins in neurodegenerative diseases: therapeutic targets and strategies. Exp Mol Med. 2015;47:e147-e147.

[19] Sneha P, Raj AK, A. The brain's structural connectome mediates the relationship between regional neuroimaging biomarkers in Alzheimer's disease. J Alzheimers Dis. 2017;55(4):1639-1657.

[20] Takata K, Kitamura Y, Tsuchiya D, Kawasaki T, Taniguchi T, Shimohama S. High mobility group box protein-1 inhibits microglial $\mathrm{A} \beta$ clearance and enhances $\mathrm{A} \beta$ neurotoxicity. J Neurosci Res. 2004;78:880-891.

[21] Barbash S, Soreq H. Threshold-independent meta-analysis of Alzheimer's disease transcriptomes shows progressive changes in hippocampal functions, epigenetics and microRNA regulation. Curr Alzheimer Res. 2012;9(4):425-435.

[22] Ma XB. Research on the structure and functions of antimicrobial peptides. Journal of Anhui Agricultural Science. 2009;37(23):10878-10880.

[23] S C. Advances in peptide pharmaceuticals. Curr Pharm Biotechnol. 2009;10(1):122-137.

[24] Zheng L, Dong HZ, Zhao MM, Zhao YJ, Su GW. Structure-activity relationship of antioxidant dipeptides: dominant role of Tyr, Trp, Cys and Met residues. J Funct Foods. 2016;21:485-496.

[25] Sabine L, Kerry C, Gabriella F, David O, Bartlomiej T, Joanna G. The ageing brain: effects on DNA repair and DNA methylation in mice. Genes. 2017;8(2):75-75.

[26] Vladimir VS, Aaron RF, Dan ZM, O J. Blood-brain barrier dysfunction in aging induces hyper-activation of TGF- $\beta$ signaling and chronic yet reversible neural dysfunction. Sci Transl Med. 2019;p. 8954-8954.

[27] Zhu SS, Zhang L, Dai YL, Zhang Y, Xu Y, Shi XP. A bioinformatics analysis of differentially expressed genes in Alzheimer's disease. Journal of Chongqing Medical University. 2019;44:4-4.

[28] Jager D, Srivastava PL, Lunnon G, Burgess K, Schalkwyk J, Yu LC, et al. Alzheimer's disease pathology is associated with early alterations in brain DNA methylation at ANK1, BIN1, RHBDF2 and other loci. Nat Neurosci. 2014;17(9):1156-1163.

[29] Marques S, Lemos R, Ferreiro E, Martins M, Mendoca A, Santana I. Epigenetic regulation of BACE1 in Alzheimer's disease patients and in transgenic mice. Neuroscience. 2012;220(6):256-266.

[30] Wan G, Zhou W, Hu Y, Ma R, Liu JS, G. Transcriptional regulation of lncRNA genes by histone modification in Alzheimer's disease. Biomed Res Int. 2016;3164238:1-4.

[31] Lin PI, Tai YT, Chan WP, Lin YL, Liao MH, Chen RM. Estrogen/ER $\alpha$ signaling axis participates in osteoblast maturation via upregulating chromosomal and mitochondrial complex gene expressions. Oncotarget. 2017;9(1):1169-1186. 
[32] Kang S, Byun J, Son SM, Jung MI. Thrombospondin1 protects against $\mathrm{A} \beta$-induced mitochondrial fragmentation and dysfunction in hippocampal cells. Cell Death Discov. 2018;4(1):31-31.

[33] Ingolia NT, Ghaemmaghami S, Newman JR, Weissman JS. Genome-wide analysis in vivo of translation with nucleotide resolution using ribosome profling. Science. 2009;324(5924):218223.

[34] Lei N, Gang W, Brockman FJ, Zhang WW. Integrated analysis of transcriptomic and proteomic data of desulfovibrio vulgaris: zero-inflated Poisson regression models to predict abundance of undetected proteins. Bioinformatics. 2006;22(13):1641-1647.

[35] Wandaliz TG, Zhang WW, Runger GC, Johnson R, Meldrum DR. Integrative analysis of transcriptomic and proteomic data of desulfovibrio vulgaris: a non-linear model to predict abundance of undetected proteins. Bioinformatics. 2009;25(15):1905-1914.

[36] Humphries C, Gilbert J, Kohli M, Whitehead P, Mash D, Beecham $\mathrm{G}$, et al. RNA transcription and editing in Alzheimer's disease. Alzheimers Dement. 2013;9(4):P829-P830. 